\title{
LAPTM4B* 2 allele is associated with the development of papillary thyroid carcinoma in Chinese women
}

\author{
YUE MENG $^{1}$, ROULI ZHOU ${ }^{2}$, JIANJUN XU $^{1}$ and QINGYUN ZHANG ${ }^{1}$ \\ ${ }^{1}$ Department of Clinical Laboratory, Key Laboratory of Carcinogenesis and Translational Research (Ministry of Education), \\ Peking University Cancer Hospital and Institute, Beijing 100142; ${ }^{2}$ Department of Cell Biology, \\ School of Basic Medical Sciences, Peking University, Beijing 100083, P.R. China
}

Received December 12, 2015; Accepted May 11, 2017

DOI: $10.3892 / \mathrm{ol} .2017 .6503$

\begin{abstract}
Lysosome-associated protein transmembrane 4- $\beta$ (LAPTM4B) contains a polymorphic region that contributes to the increased risk of numerous types of tumor. However, no study has yet demonstrated an association between the expression of the LAPTM4B gene and tumor differentiation, and the reason that LAPTM4B polymorphisms affect the susceptibility of individuals to cancer remains to be elucidated. The present study assessed the possible association between LAPTM4B polymorphism and the risk of papillary thyroid carcinoma (PTC), and attempted to identify the underlying mechanism for variation in patient susceptibility with respect to transcription regulation in the polymorphism region. The case control study included 183 papillary thyroid carcinoma patients (132 females, 51 males) and 697 healthy controls (249 females, 448 males). Genomic DNA was extracted from the blood and the genotype of LAPTM4B was identified using polymerase chain reaction (PCR). The results of the PCR revealed that LAPTM4B allele *2 was associated with PTC risk in comparison with LAPTM4B allele *1 [odds ratio (OR), 1.968; 95\% confidence interval (CI), 1.363-2.841, $\mathrm{P}<0.001]$ in females, while LAPTM4B*2 was not associated with PTC risk in males (OR, 0.996, 95\% CI, 0.615-1.612, P=0.986). Notably, LAPTM4B polymorphism was not associated with clinical parameters in the female patient group. In addition, by performing a luciferase
\end{abstract}

Correspondence to: Dr Qingyun Zhang, Department of Clinical Laboratory, Key Laboratory of Carcinogenesis and Translational Research (Ministry of Education), Peking University Cancer Hospital and Institute, 52 Fucheng Road, Haidian, Beijing 100142, P.R. China

E-mail: zhqy_208@163.com

Abbreviations: LAPTM4B, lysosomal protein transmembrane 4- $\beta$; PTC, papillary thyroid carcinoma; ORs, odd ratios; CI, confidence interval; BMI, body mass index

Key words: lysosome-associated protein transmembrane $4-\beta$, polymorphism, papillary thyroid carcinoma, transcription activity, susceptibility reporter assay in the PTC TPC1 and B-CPAP cell lines, the transcriptional activity of the $+10 /+311$ plasmid, representing LAPTM4B* 2 was reduced compared with that of the $+10 /+292$ plasmid representing LAPTM4B*1. In conclusion, the results of the present study suggested that LAPTM4B*2 was a susceptibility factor for PTC in the female Chinese population and this may not be caused by the transcriptional regulation of LAPTM4B polymorphism region in TPC1 and B-CPAP cell lines.

\section{Introduction}

The incidence of thyroid carcinoma has increased rapidly over the past 3 decades (1). However, the reason for this increase in incidence remains to be elucidated (2). Certain studies have reported that this increase may be due to the high detection rate resulting from the increasing prevalence of thyroid ultrasonography and subsequent fine-needle aspiration cytology (3-5). A previous study suggested that the number of teenagers $(<20$ years) with the disease, in which screening for thyroid carcinoma is not performed, is increasing (6). Papillary thyroid carcinoma (PTC) is the primary subtype of thyroid cancer that has contributed to this increased incidence globally. The etiological factors for the development of papillary thyroid carcinoma include exogenous factors, including radiation exposure, high iodine intake, Hashimoto's thyroiditis and obesity, and endogenous factors, such as certain gene mutations that have been demonstrated to serve a key role in progression of thyroid tumorigenesis (7-9). The incidence of PTC in females is more than twice than in males, which may be due to the fact that estrogen may promote the proliferation of thyroid carcinoma cells (10). Although there have been a number of hypotheses regarding thyroid tumorigenesis, further study is required.

Lysosomal protein transmembrane $4-\beta$ (LAPTM4B) is typically highly expressed in hepatocellular carcinoma in comparison with normal liver cells (11). LAPTM4B is located on chromosome 8q22.1 and includes 7 exons and 6 introns (12). In total two alleles of LAPTM4B have been identified, termed LAPTM4B*1 allele and LAPTM4B*2 allele (12). The difference between these alleles is that LAPTM4B* 2 has an extra 19 base pairs (bp) sequence arranged in tandem at the 5'untranslated region (UTR) in exon 1 (12). The two alleles generate 
three potential genotypes: LAPTM4B*1/1; LAPTM4B*1/2; LAPTM4B $2 / 2$ (12).

Previous studies have established that LAPTM4B gene polymorphism is associated with tumor susceptibility; notably, LAPTM4B*2 is associated with a high risk of breast (13), stomach (14), lung (15), colon (16), endometrial (17), cervical (18), gall bladder (19), ovarian cancer and melanoma $(20,21)$. Nevertheless, no significant difference in the frequency of LAPTM4B" 2 is observed in patients with nasopharyngeal carcinoma and esophageal carcinoma, compared with corresponding controls $(16,22)$. These results suggest that LAPTM4B* 2 may be specifically associated with susceptibility to certain tumors. Papillary thyroid carcinoma is a well-differentiated carcinoma that has not yet been associated with LAPTM4B gene polymorphism. Therefore, in the present study, a case-control assay was performed to assess LAPTM4B gene polymorphism association with susceptibility to PTC in the female Chinese population. A dual-luciferase reporter assay was also performed in two types of thyroid papillary cells to assess the transcriptional activity of LAPTM4B*1 compared with LAPTM4B*2.

\section{Materials and methods}

Patients and tissue samples. Blood samples were collected in EDTA-containing tubes from 183 patients with PTC who underwent surgical resection at Beijing Cancer Hospital Affiliated with Peking University (Beijing, China) from April 2015 to September 2015 and frozen at $-20^{\circ} \mathrm{C}$. Each patient provided informed consent for participation in the present study. Tumor-Node-Metastasis (TNM) staging was performed by Yue Meng for each patient following standard TNM guidelines (23). Clinicopathological characteristics that were evaluated included sex, age, tumor size, tumor location, body mass index (BMI), tumor invasive condition and central lymph node status. The clinicopathological features of female patients also included menopause and the number of pregnancies. The data of healthy control patients was obtained from previously published studies by Cheng et al (16) and Liu et al (14) and the consent for these data was obtained at the time of initial data retrieval.

DNA extraction and polymerase chain reaction (PCR). Frozen blood samples were thawed in a water bath at room temperature and genomic DNA was extracted from $600 \mu \mathrm{l}$ of each blood sample using a DNA extraction kit (Tiangen Biotech Co., Beijing, China; cat. no., DP304-03), according to the manufacturer's protocol. For each sample, the genotype of LAPTM4B was identified by PCR, using the following primers: forward, 5'-GCCGACTAGGGGACTGGCGGA-3'; and reverse, 5'-CGAGAGCTCCGAGCTTCTGCC-3'. The PCR mixture $(25 \mu \mathrm{l})$ contained 2X PCR Taq mix $(12.5 \mu \mathrm{l}$; KT-201; Tiangen Biotech Co., Beijing, China), $1 \mu$ l template DNA at final concentration $100 \mathrm{ng} / \mu 1,0.5 \mu 1 \beta$-actin forward primer at a final concentration $5 \mu \mathrm{M}, 0.5 \mu \mathrm{l} \beta$-actin reverse primer at a final concentration $5 \mu \mathrm{M}, 1 \mu \mathrm{l}$ LAPTM4B forward primer at a final concentration $10 \mu \mathrm{M}, 1 \mu \mathrm{l}$ LAPTM4B reverse primer at a final concentration $10 \mu \mathrm{M}$ and $9.5 \mu \mathrm{lddH} 2 \mathrm{O} .0 .5 \mathrm{U}$ Taq polymerase (Tiangen Biotech Co.) and $1 \mu 1$ template DNA at a final concentration $100 \mathrm{ng} / \mu \mathrm{l}$. Human $\beta$-actin was used
Table I. General characterization of case and control group.

\begin{tabular}{lccc}
\hline & $\begin{array}{c}\text { Controls, } \\
\mathrm{n}(\%) \\
\mathrm{n}=697\end{array}$ & $\begin{array}{c}\text { Thyroid } \\
\text { carcinoma, } \mathrm{n}(\%) \\
\mathrm{n}=183\end{array}$ & \\
Characteristics & P-value $^{\mathrm{a}}$ \\
\hline Sex & & & $\mathrm{P}<0.001$ \\
Female & $249(35.7)$ & $132(72.1)$ & \\
Male & $448(64.2)$ & $51(27.8)$ & \\
Age, years & & & $\mathrm{P}<0.001$ \\
$\leq 40$ & $110(15.8)$ & $87(47.5)$ & \\
$41-50$ & $223(32.0)$ & $49(26.8)$ & \\
$51-60$ & $142(20.4)$ & $31(16.9)$ & \\
$61-70$ & $106(15.2)$ & $15(8.2)$ & \\
$>70$ & $116(16.6)$ & $1(0.6)$ & \\
& &
\end{tabular}

${ }^{\mathrm{a}}$ Analyzed using a $\chi^{2}$ test.

as an internal positive control using the following primers: Forward, 5'-TCACCAACTGGGACGACAT-3'; and reverse, 5'-AGGTAGTCAGTCAGGTCCCG-3'. The PCR conditions were $95^{\circ} \mathrm{C}$ denaturation for $5 \mathrm{~min}, 30$ cycles of $30 \mathrm{sec}$ each at $94^{\circ} \mathrm{C}, 30 \mathrm{sec}$ at $64.1^{\circ} \mathrm{C}$ and $30 \mathrm{sec}$ at $72^{\circ} \mathrm{C}$, followed by extension at $72^{\circ} \mathrm{C}$ for $5 \mathrm{~min}$. The PCR products were analyzed by electrophoresis using a $2.5 \%$ agarose gel and visualized with ethidium bromide. The LAPTM4B*1/1 genotype produced a 204 bp band, the LAPTM4B*2/2 genotype produced a $223 \mathrm{bp}$ band. The LAPTM4B*1/2 genotype produced 204 and 223-bp bands. The products of PCR were sent to SBS Genetech Co., Ltd (Beijing, China) for sequencing.

Cells and culture conditions. Human PTC TPC-1 and B-CPAP cell lines, were maintained in RPMI-1640 medium (Gibco; Thermo Fisher Scientific, Inc., Waltham, MA, USA) supplemented with $10 \%$ fetal bovine serum (Gibco; Thermo Fisher Scientific, Inc.) and penicillin streptomycin solution (cat. no., SV30010; HyClone; GE Healthcare Life Sciences, Little Chalfont, UK) at $1 \mathrm{X}$ working concentration. The cells were cultured at $37^{\circ} \mathrm{C}$ in a $5 \% \mathrm{CO}_{2}$ atmosphere. The cells were obtained from the Type Culture Collection of the Chinese Academy of Sciences (Shanghai, China).

Transient transfection and dual luciferase reporter assay. The PTC cells were seeded into 24 -well plates $\left(1 \times 10^{5}\right.$ cells/well), and incubated for $12 \mathrm{~h}$ at $37^{\circ} \mathrm{C}$ in complete medium. The TPC1 and B-CPAP cells were transfected with $0.5 \mu \mathrm{g}$ of PGL-3 reporter constructs with a number of LAPTM4B promoter inserts and pGL-3 promoter vector; all truncated plasmids were conserved by the laboratory [Key Laboratory of Carcinogenesis and Translational Research (Ministry of Education), Department of Clinical Laboratory, Peking University Cancer Hospital \& Institute, Beijing, China] (24). Different regions of LAPTM4B promoter were inserted to PGL-3 reporter constructs: $-1341 /+191,-881 /+191,-558 /+191$, $-206 /+174,-38 /+191,+1-/+292$ and $+10 /+311$. A $0.02-\mu \mathrm{g}$ phRL-CMV plasmid (E6271; Promega Corporation, Madison, WI, USA) was transfected in each well at the same time to 
Table II. Genotype and allelic frequencies of LAPTM4B gene polymorphism in PTC patients and controls.

\begin{tabular}{lccc}
\hline $\begin{array}{l}\text { LAPTM4B } \\
\text { status }\end{array}$ & $\begin{array}{c}\text { Healthy controls } \\
\mathrm{n}=697\end{array}$ & $\begin{array}{c}\text { PTC, } \mathrm{n}(\%) \\
\mathrm{n}=183\end{array}$ & $\begin{array}{c}\text { Odds ratio }^{\mathrm{b}} \\
(95 \% \text { CI })\end{array}$ \\
\hline $\begin{array}{l}\text { Allele } \\
* 1\end{array}$ & $1,058(75.9)$ & $253(69.1)$ & \\
${ }^{*} 2$ & $336(24.1)$ & $113(30.9)$ & $1.516(1.140-2.017)$ \\
Genotype & $397(57.0)$ & & \\
${ }^{*} 1 / 1$ & $264(37.8)$ & $90(49.2)$ & $1.352(0.919-1.987)$ \\
${ }^{*} 1 / 2$ & $36(5.2)$ & $73(39.9)$ & $2.765(1.384-5.524)$ \\
$* 2 / 2$ & $20(10.9)$ & 0.125 \\
\hline
\end{tabular}

${ }^{a}$ Data were analyzed by non-conditional logistic regression analysis. ${ }^{\mathrm{b}}$ Adjusted for age and sex. LAPTM4B, lysosome-associated protein transmembrane $4-\beta$; PTC, papillary thyroid carcinoma; CI, confidence interval.

Table III. Association between LAPTM4B genotypes and clinical characteristics of papillary thyroid carcinoma.

\begin{tabular}{|c|c|c|c|c|}
\hline \multirow[b]{2}{*}{ Characteristics } & \multicolumn{3}{|c|}{ LAPTM4B genotype } & \multirow[b]{2}{*}{ P-value } \\
\hline & $1 / 1$ & $1 / 2$ & $2 / 2$ & \\
\hline Age, years & & & & 0.705 \\
\hline$>40$ & 50 & 37 & 12 & \\
\hline$\leq 40$ & 40 & 36 & 8 & \\
\hline Sex & & & & 0.046 \\
\hline Female & 64 & 49 & 19 & \\
\hline Male & 26 & 24 & 1 & \\
\hline CLNM & & & & 0.219 \\
\hline Positive & 56 & 53 & 11 & \\
\hline Negative & 34 & 20 & 9 & \\
\hline Invasive tumor & & & & 0.531 \\
\hline Yes & 49 & 39 & 8 & \\
\hline No & 41 & 34 & 12 & \\
\hline Tumor size, $\mathrm{cm}$ & & & & 0.836 \\
\hline$\geq 1$ & 40 & 26 & 8 & \\
\hline$<1$ & 46 & 42 & 11 & \\
\hline Unknown & 4 & 5 & 1 & \\
\hline TNM stage & & & & 0.290 \\
\hline I-II & 58 & 41 & 10 & \\
\hline III-IV & 19 & 16 & 8 & \\
\hline Unknown & 13 & 16 & 2 & \\
\hline Tumor location & & & & 0.777 \\
\hline One side & 17 & 8 & 4 & \\
\hline Two sides & 1 & 3 & 0 & \\
\hline Isthmus & 3 & 2 & 1 & \\
\hline Two sides and isthmus & 7 & 7 & 1 & \\
\hline One side and isthmus & 62 & 53 & 14 & \\
\hline BMI & & & & 0.531 \\
\hline$>25$ & 49 & 39 & 8 & \\
\hline$\leq 25$ & 41 & 34 & 12 & \\
\hline
\end{tabular}

LAPTM4B, lysosome-associated protein transmembrane 4- $\beta$; CLNM, central lymph node metastasis; TNM, tumor-node-metastasis.

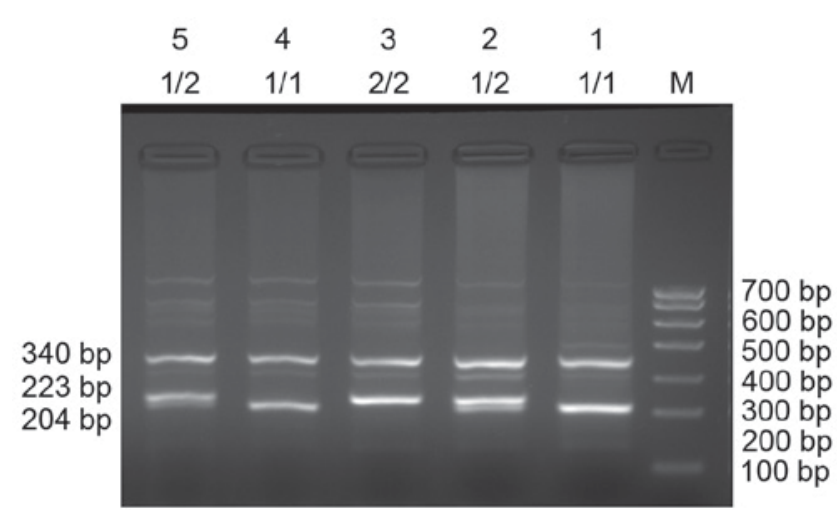

Figure 1. Genotyping of LAPTM4B alleles in 5 patient samples. Lanes 1 and 4, LAPTM4B*1/1; lanes 2 and 5, LAPTM4B*1/2; lane 3, LAPTM4B*2/2. M, DNA marker; LAPTM4B, lysosome-associated protein transmembrane $4-\beta ;$ Bp, base pairs.

normalize for the transfection efficiency. Transfection was performed using Lipofectamine 3000 transfection reagent (Invitrogen; Thermo Fisher Scientific, Inc.) according to the manufacturer's protocol. At $48 \mathrm{~h}$ following transfection, cell lysates were collected for luciferase activity detection using the DualGlo Luciferase Assay system (Promega Corporation), according to the manufacturer's protocol. All reactions were performed in triplicate and results were normalized to the Renilla luciferase activity. The luciferase value of all the plasmids was compared with that of the pGL-3 promoter (positive control), which was set as $100 \%$.

Statistics analysis. All data were analyzed using SPSS v22.0 software (IBM Corp., Armonk, NY, USA). Genotypic frequencies were tested for Hardy-Weinberg equilibrium using the $\chi^{2}$ test. Fisher's exact test was used to calculate the genotypic frequency and other parametric distributions between cancer cases and controls. Associations between genotypes and the risk of thyroid papillary cancer were estimated using odds ratios (ORs) and 95\% confidence intervals (CIs), which were computed by using unconditional logistic regression models. All ORs were adjusted for age and sex, as appropriate. A one-way analysis of variance was applied for the comparison 

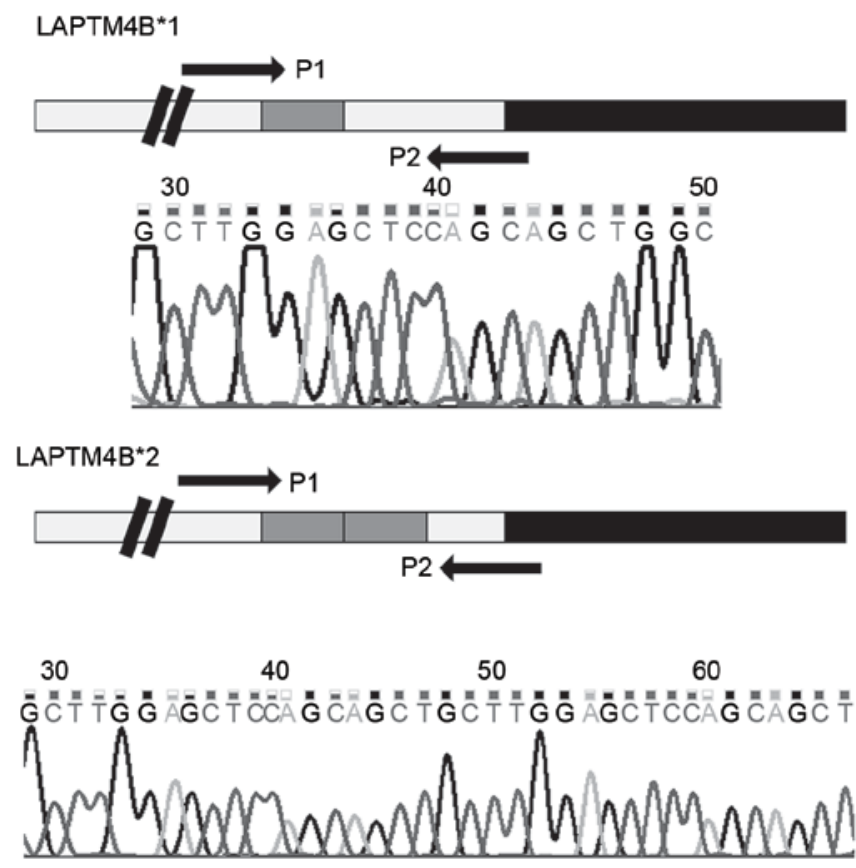

Figure 2. Sequence of polymerase chain reaction products form patient blood samples. LAPTM4B* 1 contains one 19 bp sequence (gray box), located in the promoter region (white box), following the transcription start site (double black lines) and prior to the open reading frame region (black box). LAPTM4B 2 contains two 19 bp sequences located at the same position. Sequencing results are presented below the gene structure display. LAPTM4B, lysosome-associated protein transmembrane $4-\beta$.

of luciferase report assay data, followed by a least significant difference (LSD) multiple-range post-hoc test to detect significant differences between different groups. $\mathrm{P}<0.05$ was considered to indicate a statistically significant difference.

\section{Results}

LAPTM4B genotypes. The three different genotypes were identified using PCR: LAPTM4B*1/1, homozygotes; LAPTM4B*2/2, homozygotes; and LAPTM4B*1/2 heterozygotes (Fig. 1). The LAPTM4B*1/1 genotype was identified by the presence of a 204-bp band, the LAPTM4B*2/2 genotype was identified by the presence of a 223-bp band. The presence of 204-bp and 223-bp bands together identified the heterozygotic LAPTM4B*1/2 genotype. The human $\beta$-actin PCR product was a 340-bp band that appeared in all internal controls. PCR products were sequenced and the results are presented in Fig. 2.

LAPTM4B genotypes and PTC susceptibility. The frequency of LAPTM4B genotypes of 183 patients $(\mathrm{P}=0.37)$ and 697 health controls $(\mathrm{P}=0.352)$ were in accordance with Hardy-Weinberg equilibrium distribution. There were statistically significant differences in age and sex between patients with PTC and healthy controls in the present study (Table I). Following OR adjustments by sex and age, the risk of PTC in patients with the LAPTM4B*2/2 genotype was identified to be 2.765-fold higher compared with that in samples with the LAPTM4B ${ }^{*} 1 / 1$ genotype. The risk of PTC in patients with a copy of LAPTM4B 2 is 1.516 -fold higher compared with that in patients with only LAPTM4B*1 (Table II).

Genotypes of LAPTM4B and clinical parameters. By analyzing the clinical parameters of patients with PTC and healthy controls, the female sex was identified to be significantly associated with LAPTM4B 2/2 genotype, whereas age, central lymph node metastasis condition, invasive condition of the tumor, tumor size, BMI, tumor location and TMN stage were not significantly associated with a specific LAPTM4B genotype (Table III).

LAPTM4B genotypes and PTC susceptibility compared between females and males. As sex was associated with a certain LAPTM4B genotype, the LAPTM4B genotypes of females and males were analyzed separately. In the female patients that were analyzed, a LAPTM4B*2/2 genotype increased the risk of developing PTC 5.494-fold compared with the LAPTM4B*1/1 genotype. A female carrying a copy of LAPTM4B*2 had a 1.968-fold increased risk of PTC compared with those carrying only copies of LAPTM4B*1 alone (Table IV). However, no statistically significant difference in LAPTM4B genotype frequency was exhibited between the PTC patients and the healthy controls in males (Table V).

Association between LAPTVM4B genotype and clinical parameters in female patients. The clinical parameters and genotypes of LAPTM4B were further analyzed in the female participants. There was no significant association between age, central lymph node metastasis, invasive condition of the tumor, tumor size, TNM stage, tumor location, BMI, number of pregnancies or menopause condition and LAPTM4B genotype (Table VI).

Dual luciferase reporter assay. The difference between LAPTM4B*1 and LAPTM4B*2 is the presence of a 19-bp sequence located in the 5'UTR of exon 1. Thus, the current study hypothesized that the extra 19-bp sequence may affect the transcriptional activity of LAPTM4B* 2 , resulting in an 
Table IV. Frequency of LAPTM4B in female PTC patients and healthy controls.

\begin{tabular}{lccc}
\hline $\begin{array}{l}\text { LAPTM4B } \\
\text { status }\end{array}$ & $\begin{array}{c}\text { Healthy controls, } \mathrm{n}(\%) \\
\mathrm{n}=249\end{array}$ & $\begin{array}{c}\text { PTC, } \mathrm{n}(\%) \\
\mathrm{n}=132\end{array}$ & $\begin{array}{c}\text { Odds ratio } \\
(95 \% \text { CI })\end{array}$ \\
\hline $\begin{array}{l}\text { Allele } \\
{ }^{*} 1\end{array}$ & $395(79.3)$ & $177(67.0)$ & \\
${ }^{*} 2$ & $103(20.7)$ & $87(33.0)$ & $1.968(1.363-2.841)$ \\
Genotype & $156(62.7)$ & $64(48.5)$ & \\
${ }^{*} 1 / 1$ & $83(33.3)$ & $49(37.1)$ & $1.443(0.880-2.365)$ \\
${ }^{*} 1 / 2$ & $10(4.0)$ & $19(14.4)$ & $5.494(2.198-13.730)$ \\
${ }^{*} 2 / 2$ & & & $0.146^{\mathrm{b}}$ \\
\hline
\end{tabular}

${ }^{a}$ Compared with LAPTM4B ${ }^{*} 1 .{ }^{\text {b } C o m p a r e d ~ w i t h ~ L A P T M 4 B ~}{ }^{*} 1 / 1$. LAPTM4B, lysosome-associated protein transmembrane 4- $\beta$; PTC, papillary thyroid carcinoma; CI, confidence interval.

Table V. The frequency of LAPTM4B in male papillary thyroid carcinoma patients and male healthy controls.

\begin{tabular}{lccc}
\hline $\begin{array}{l}\text { LAPTM4B } \\
\text { status }\end{array}$ & $\begin{array}{c}\text { Healthy controls, } \mathrm{n}(\%) \\
\mathrm{n}=448\end{array}$ & $\begin{array}{c}\text { PTC, } \mathrm{n}(\%) \\
\mathrm{n}=51\end{array}$ & OR (95\% CI) \\
\hline $\begin{array}{c}\text { Allele } \\
* 1\end{array}$ & $663(74.0)$ & $76(74.5)$ & \\
$* 2$ & $233(26.0)$ & $26(25.5)$ & $0.996(0.615-1.612)$ \\
Genotype & $241(53.8)$ & $26(50.9)$ & $0.986^{\mathrm{a}}$ \\
$* 1 / 1$ & $181(40.4)$ & $24(47.1)$ & $1.187(0.649-2.169)$ \\
$* 1 / 2$ & $26(5.8)$ & $1(2.0)$ & $0.445(0.057-3.481)$ \\
$* 2 / 2$ & & & $0.577^{\mathrm{b}}$ \\
\hline
\end{tabular}

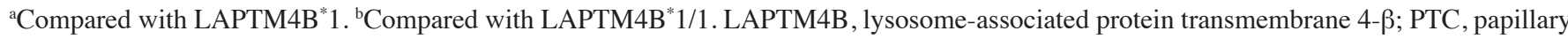
thyroid carcinoma; CI, confidence interval.

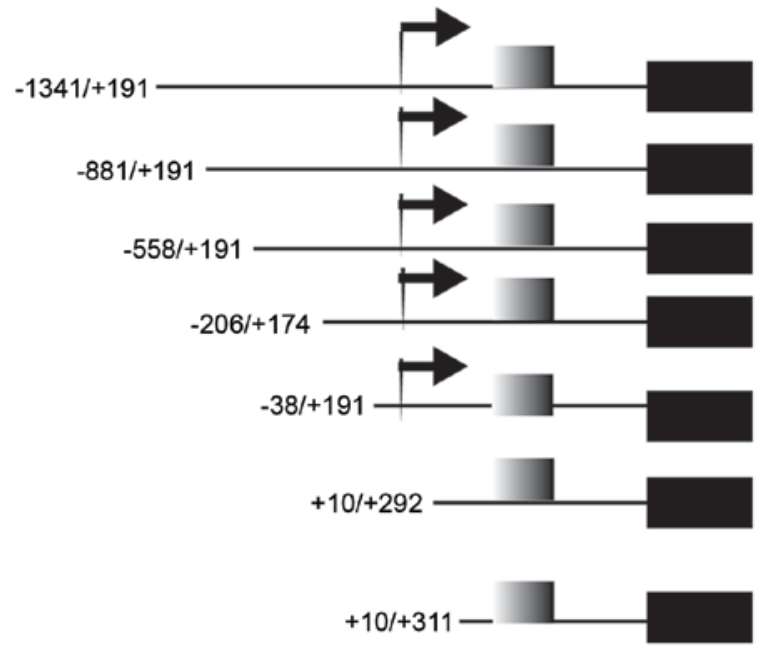

Figure 3. Plasmid details of those used in the dual luciferase reporter assay. The black box represents the luciferase gene; the gray box represents the 19 bp sequence; the arrow represents the transcription start site.

altered biological function that causes the patients that carry LAPTM4B*2 2 to be susceptible to PTC. In total seven types of luciferase reporter plasmid were transfected into TPC1 and
B-CPAP cells (Fig. 3). The $+10 /+292$ plasmid, which contains one 19-bp sequence, had the highest transcription activity, and the $+10 /+311$ plasmid, including an extra 19-bp sequence, had the second highest (Fig. 4). It is possible that there may be transcription factors that bind to the extra 19-bp sequence, increasing transcriptional activity; however, the susceptibility of individuals with the LAPTM4B* 2 to PTC is not caused by transcriptional regulation of the polymorphism region in these two cell lines.

\section{Discussion}

PTC is the most frequently occurring well-differentiated form of thyroid carcinoma (25). In recent years, PTC has become the most common type of cancer in female patients (26). Gene mutation, rearrangement and certain genetic polymorphisms are hypothesized to be the etiological factors associated with the development of PTC (27-29). In addition, female hormones may also promote susceptibility to PTC in females (30). However, the etiology of PTC remains to be elucidated. The present study has demonstrated that LAPTM4B gene polymorphism is a susceptibility factor to papillary thyroid carcinoma in Chinese females. A female patient carrying a copy of LAPTM4B* 2 is more susceptible to PTC compared with those carrying only LAPTM4B* 1 . 
Table VI. Association between LAPTM4B genotypes and clinical characteristics of papillary thyroid carcinoma in the female group.

\begin{tabular}{|c|c|c|c|c|}
\hline \multirow[b]{2}{*}{ Characteristics } & \multicolumn{3}{|c|}{ LAPTM4B genotype } & \multirow[b]{2}{*}{ P-value } \\
\hline & *1/1 (n=64) & $* 1 / 2(n=49)$ & $* 2 / 2(n=19)$ & \\
\hline Age, years & & & & 0.745 \\
\hline$>40$ & 34 & 27 & 12 & \\
\hline$\leq 40$ & 30 & 22 & 7 & \\
\hline CLNM $^{\mathrm{a}}$ & & & & 0.084 \\
\hline Positive & 21 & 10 & 9 & \\
\hline Negative & 43 & 39 & 10 & \\
\hline Invasive tumor & & & & 0.640 \\
\hline Yes & 28 & 24 & 7 & \\
\hline No & 36 & 25 & 12 & \\
\hline Tumor size, $\mathrm{cm}$ & & & & 0.816 \\
\hline$\geq 1$ & 28 & 16 & 7 & \\
\hline$<1$ & 33 & 31 & 11 & \\
\hline Unknown & 3 & 2 & 1 & \\
\hline TNM stage & & & & 0.228 \\
\hline I-II & 41 & 27 & 9 & \\
\hline III-IV & 16 & 11 & 8 & \\
\hline Unknown & 7 & 11 & 2 & \\
\hline Tumor location & & & & 0.856 \\
\hline One side & 10 & 6 & 4 & \\
\hline Two sides & 0 & 2 & 0 & \\
\hline Isthmus & 2 & 2 & 1 & \\
\hline Two sides and isthmus & 4 & 3 & 1 & \\
\hline One side and isthmus & 48 & 36 & 13 & \\
\hline BMI & & & & 0.64 \\
\hline$>25$ & 28 & 24 & 7 & \\
\hline$\leq 25$ & 36 & 25 & 12 & \\
\hline Pregnancies & & & & 0.852 \\
\hline 0 & 12 & 10 & 4 & \\
\hline 1 & 37 & 32 & 14 & \\
\hline$\geq 2$ & 10 & 6 & 1 & \\
\hline Unknown & 5 & 1 & 0 & \\
\hline Menopause & & & & 0.138 \\
\hline Yes & 16 & 12 & 9 & \\
\hline No & 48 & 37 & 10 & \\
\hline
\end{tabular}

CLNM, central lymph node metastasis; TNM, tumor-node-metastasis; BMI, body mass index.

In the present study, a dual luciferase reporter assay was performed, demonstrating that the transcriptional activity of LAPTM4B*1 was higher compared with that of LAPTM4B*2 in PTC cells. Previous clinical studies have revealed that the overexpression of LAPTM4B gene facilitates tumorigenesis by promoting cell proliferation (31-33), inhibiting apoptosis (34) and initiating autophagy (35) in numerous types of carcinoma. Therefore, the current study hypothesized that LAPTM4B*2 may have an increased transcriptional activity compared with that LAPTM4B*1, which may explain why people with
LAPTM4B allele ${ }^{*} 2$ demonstrate increased susceptibility to PTC. However, the transcription activity of LAPTM4B* 2 was reduced compared with that of LAPTM4B*1 in PTC cells. Therefore, the underlying mechanism as to why patients with LAPTM4B*2 are more susceptible to PTC is not associated with the transcriptional regulation of the polymorphism region in TPC1 and B-CPAP cell lines.

Screening the sequence of the LAPTM4B*2 and LAPTM4B* 1 , the extra 19 bp sequence may directly induce the skipping of the termination codon, which would promote 

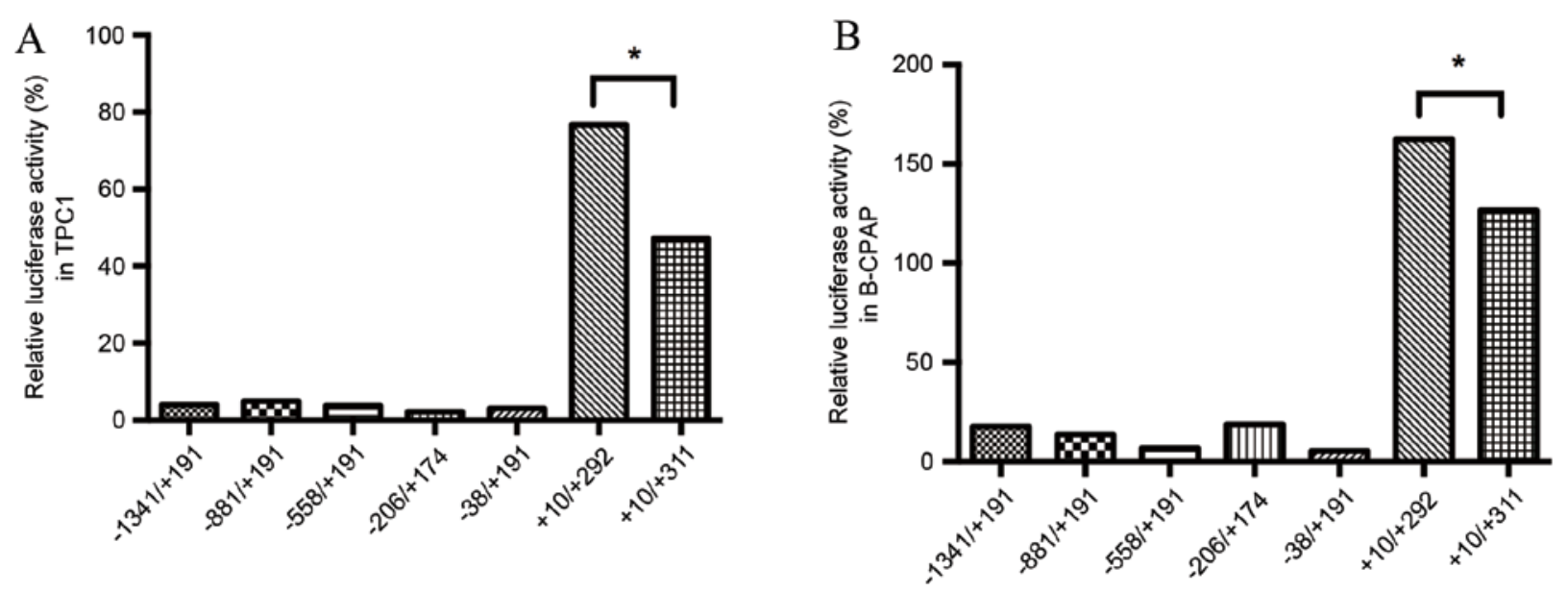

Figure 4. Relative luciferase activity in papillary thyroid carcinoma cells. These values are from three independence experiments performed in triplicate in the (A) TPC1 and (B) B-CPAP cell lines ( $\mathrm{P}<0.05 ; \mathrm{n}=3$ ).

*1 1 GAATCTCGAC CCTTGAATGG AGTTACACGA ACGGCCAGAT GAAAGAAGgA AGGCCCGGAC

*2 1 GAATCTCGAC CCTTGAATGg AGTTACACGA ACGGCCAGAT GAAAGAAGgA AGGCCCGGAC $\begin{array}{lllllllllllllllll}M & E & L & H & E & R & P & D & E & R & R & K & A & R & T & 15\end{array}$

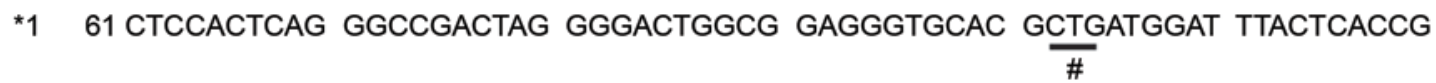

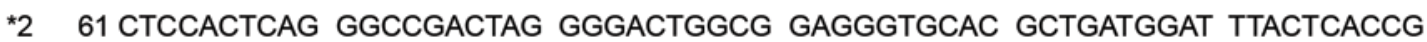

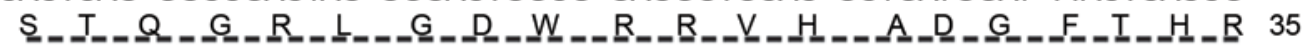

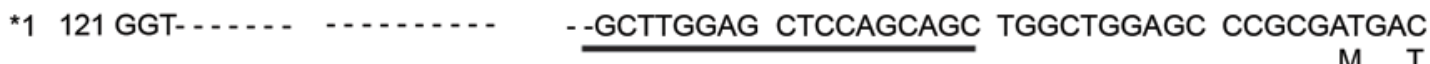

*2 121 GGTGCTTGGA GCTCCAGCAG CTGCTTGGAG CTCCAGCAGC TGGCTGGAGC CCGCGATGAC

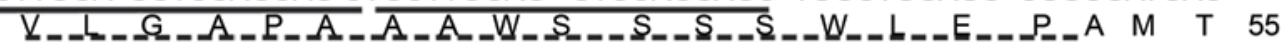

Figure 5. Comparison of the putative proteins encoded by LAPMTB4*1 and LAPTM4B*2. Partial sequences of exon 1 are demonstrated. The nucleotide sequences are numbered with the putative transcription start site designated as +1 . In-frame stop codons are underlined and marked with a hash symbol. The 19 bp sequences in both alleles are underlined. Owning to in-frame stop codons at nucleotide 40 and 103, mRNA of LAPTM4B* 1 that only begin translation at nucleotide 157 . However, LAPTM4B* 2 may initiate translation at nucleotide 17 , which would produce an extra 53 amino acids, indicated by letters underscored with the dotted line at N-terminus of LAPTM4B. LAPTM4B, lysosome-associated protein transmembrane 4- $\beta$.

the protein-code frame lengthened of 53 amino acids at the $\mathrm{N}$ terminus and produce a variation of the functional protein compared with that of LAPTM4B*1 (Fig. 5). The N terminus of the LAPTM4B protein serves a key role in cell signal transduction, ligand-receptor binding (12) and certain other fields such as cell invasion, migration and cell proliferation (36). Therefore, the protein encoded by LAPTM4B* 2 may promote the progression of tumor development.

The present study compared the LAPTM4B genotypes between PTC patients and a healthy control group. The LAPTM $4 \mathrm{~B}^{*} 2 /^{*} 2$ genotype distribution frequency and LAPTM4B 2 distribution frequency between the two groups were significantly different. However, the analysis of clinical parameters revealed that the LAPTM4B genotype frequency was associated with the female sex, so LAPTM4B genotype frequency was analyzed separately for males and females. A significant difference in LAPTM4B genotype frequency was observed between female patients with PTC and female healthy controls. This result verified that females have increased susceptibility to PTC; however, the negative results observed in the male group requires verification in a larger sample.

Previous studies have revealed that individuals with the LAPTM4B*1/22 genotype are also susceptible to numerous types of tumor $(11,12,19)$. Nevertheless, the present study into PTC indicates that only those with the LAPTM $4 B^{*} 2 /{ }^{*} 2$ genotype are more susceptible to PTC. Unlike less-differentiated tumors, which are more aggressive and impart a poorer prognosis, well-differentiated PTC may require homozygotic LAPTM $4 B^{*} 2 / 2$ to drive tumorigenesis.

In conclusion, the present study demonstrated that the presence of the LAPTM4B*2 allele is associated with increased susceptibility of Chinese females to PTC, and that this susceptibility is not caused by transcriptional regulation mediated by the 19 bp addition in LAPTM4B*2. 


\section{Acknowledgements}

This work was supported by the National Natural Science Foundation of China (No. 81572910).

\section{References}

1. Ito Y, Nikiforov YE, Schlumberger M and Vigneri R. Increasing incidence of thyroid cancer: controversies explored. Nat Rev Endocrinol 9: 178-184, 2013.

2. Siegel R, Ma J, Zou Z and Jemal A: Cancer statistics, 2014. CA Cancer J Clin 64: 9-29, 2014.

3. Aschebrook-Kilfoy B, Grogan RH, Ward MH, Kaplan E and Devesa SS: Follicular thyroid cancer incidence patterns in the United States, 1980-2009. Thyroid 23: 1015-1021, 2013.

4. Davies Land Welch H: Current thyroid cancer trends in the United States. JAMA Otolaryngol Head Neck Surg 140: 317-322, 2014.

5. Vaccarella S, Dal Maso L, Laversanne M, Bray F, Plummer M and Franceschi S: The impact of diagnostic changes on the rise in thyroid cancer incidence: A population-based study in selected high-resource countries. Thyroid 25: 1127-1136, 2015.

6. Vergamini LB, Frazier AL, Abrantes FL, Ribeiro KB and Rodriguez-Galindo C: Increase in the incidence of differentiated thyroid carcinoma in children, adolescents and young adults: A population-based study. J Pediatr 164: 1481-1485, 2014.

7. Pellegriti G, Frasca F, Regalbuto C, Squatrito S and Vigneri R: Worldwide increasing incidence of thyroid cancer: Update on epidemiology and risk factors. J Cancer Epidemiol 2013: 965212, 2013.

8. Xu L, Port M, Landi S, Gemignani F, Cipollini M, Elisei R, Goudeva L, Müller JA, Nerlich K, Pellegrini G, et al: Obesity and the risk of papillary thyroid cancer: A pooled analysis of three case-control studies. Thyroid 24: 966-974, 2014.

9. Zou M, Baitei EY, Al-Rijjal RA, Parhar RS, Al-Mohanna FA, Kimura S, Pritchard C, Binessa HA, Alzahrani AS, Al-Khalaf HH, et al: TSH overcomes Braf(V600E)-induced senescence to promote tumor progression via downregulation of p53 expression in papillary thyroid cancer. Oncogene 35: 1909-1918, 2016.

10. Chen GG, Vlantis AC, Zeng Q and van Hasselt CA: Regulation of cell growth by estrogen signaling and potential targets in thyroid cancer. Curr Cancer Drug Targets 8: 367-377, 2008.

11. Yang H, Lin M, Xiong F, Yang Y, Nie X, McNutt MA and Zhou R: Combined lysosomal protein transmembrane 4 beta-35 and argininosuccinate synthetase expression predicts clinical outcome in hepatocellular carcinoma patients. Surg Today 41: 810-817, 2011.

12. Shao GZ, Zhou RL, Zhang QY, Zhang Y, Liu JJ, Rui JA, Wei X and Ye DX: Molecular cloning and characterization of LAPTM4B, a novel gene upregulated in hepatocellular carcinoma. Oncogene 22: 5060-5069, 2003.

13. Fan M, Liu Y, Zhou R and Zhang Q: Association of LAPTM4B gene polymorphism with breast cancer susceptibility. Cancer epidemiol 36: 364-368, 2012

14. Liu Y, Zhang QY, Qian N and Zhou RL: Relationship between LAPTM4B gene polymorphism and susceptibility of gastric cancer. Ann Oncol 18: 311-316, 2007.

15. Deng LJ, Zhang QY, Liu B and Zhou RL: Relationship between LAPTM4B gene polymorphism and susceptibility of lung cancer. Beijing Da Xue Xue Bao 37: 302-305, 2005 (In Chinese).

16. Cheng XJ, Xu W, Zhang QY and Zhou RL: Relationship between LAPTM4B gene polymorphism and susceptibility of colorectal and esophageal cancers. Ann Oncol 19: 527-532, 2008.

17. Meng F, Li H, Zhou R, Luo C, Hu Y and Lou G: LAPTM4B gene polymorphism and endometrial carcinoma risk and prognosis. Biomarkers 18: 136-143, 2013.
18. Meng F, Song H, Luo C, Yin M, Xu Y, Liu H, Zhou R and Lou G: Correlation of LAPTM4B polymorphisms with cervical carcinoma. Cancer 117: 2652-2658, 2011.

19. Yang H, Zhai G, Ji X, Xiong F, Su J and McNutt MA: Correlation of LAPTM4B polymorphisms with gallbladder carcinoma susceptibility in Chinese patients. Med Oncol 29: 2809-2813, 2012.

20. Xu Y, Liu Y, Zhou R, Meng F, Gao Y, Yang S, Li X, Yang M and Lou G: LAPTM4B polymorphisms is associated with ovarian cancer susceptibility and its prognosis. Jpn J Clin Oncol 42: 413-419, 2012

21. Zhang M, Zhou R, Xu J and Zhang Q: Relationship between LAPTM4B gene polymorphism and susceptibility of malignant melanoma in Chinese patients. Transl Oncol 7: 638-643, 2014.

22. Wang B, Xu J, Zhou R and Zhang Q: Association of LAPTM4B gene polymorphism with nasopharyngeal carcinoma susceptibility in a Chinese population. Med Oncol 30: 470, 2013.

23. Cuccurullo V and Mansi L: AJCC Cancer Staging Handbook: From the AJCC Cancer Staging Manual (7th edition). European Journal of Nuclear Medicine and Molecular Imaging 38: 408-408, 2011

24. Zhang M, Xu JJ, Zhou RL and Zhang QY: cAMP responsive element binding protein-1 is a transcription factor of lysosomal-associated protein transmembrane-4 Beta in human breast cancer cells. PLoS one 8: e57520, 2013.

25. Aschebrook-Kilfoy B, Ward MH, Sabra MM and Devesa SS: Thyroid cancer incidence patterns in the United States by histologic type, 1992-2006. Thyroid 21: 125-134, 2011.

26. Chen AY, Jemal A and Ward EM: Increasing incidence of differentiated thyroid cancer in the United States, 1988-2005. Cancer 115: 3801-3807, 2009

27. Zhao J, Huang X, Yang M, Li M and Zheng J: Association between the FTOrs8050136 polymorphism and cancer risk: A meta-analysis. Fam cancer 15: 145-153, 2016.

28. Lee YC, Chung JH, Kim SK, Rhee SY, Chon S, Oh SJ, Hong IK and Eun YG: Association between interleukin 17/interleukin 17 receptor gene polymorphisms and papillary thyroid cancer in Korean population. Cytokine 71: 283-288, 2015.

29. Soares P, Trovisco V, Rocha AS, Lima J, Castro P, Preto A, Máximo V, Botelho T, Seruca R and Sobrinho-Simões M: BRAF mutations and RET/PTC rearrangements are alternative events in the etiopathogenesis of PTC. Oncogene 22: 4578-4580, 2003.

30. Horn-Ross PL, Canchola AJ, Ma H, Reynolds P and Bernstein L: Hormonal factors and the risk of papillary thyroid cancer in the California Teachers Study cohort. Cancer Epidemiol Biomarkers Prev 20: 1751-1759, 2011.

31. Milkereit R, Persaud A, Vanoaica L, Guetg A, Verrey F and Rotin D: LAPTM4b recruits the LAT1-4F2hc Leu transporter to lysosomes and promotes mTORC1 activation. Nat commun 6: $7250,2015$.

32. Meng F, Chen X, Song H and Lou G: LAPTM4B down regulation inhibits the proliferation, invasion and angiogenesis of HeLa cells in vitro. Cell Physiol Biochem 37: 890-900, 2015.

33. Zhou L, He XD, Yu JC, Zhou RL, Yang H, Qu Q and Rui JA: Overexpression of LAPTM4B promotes growth of gallbladder carcinoma cells in vitro. Am J Surg 199: 515-521, 2010.

34. Zhou L, He XD, Yu JC, Zhou RL, Shan Y and Rui JA: Overexpression of LAPTM4B-35 attenuates epirubucin-induced apoptosis of gallbladder carcinoma GBC-SD cells. Surgery 150: 25-31, 2011

35. Tan X, Thapa N, Sun Y and Anderson RA: A kinase-independent role for EGF receptor in autophagy initiation. Cell 160: 145-160, 2015.

36. Liu X, Xiong F, Wei X, Yang H and Zhou R: LAPTM4B-35, a novel tetratransmembrane protein and its PPRP motif play critical roles in proliferation and metastatic potential of hepatocellular carcinoma cells. Cancer sci 100: 2335-2340, 2009. 